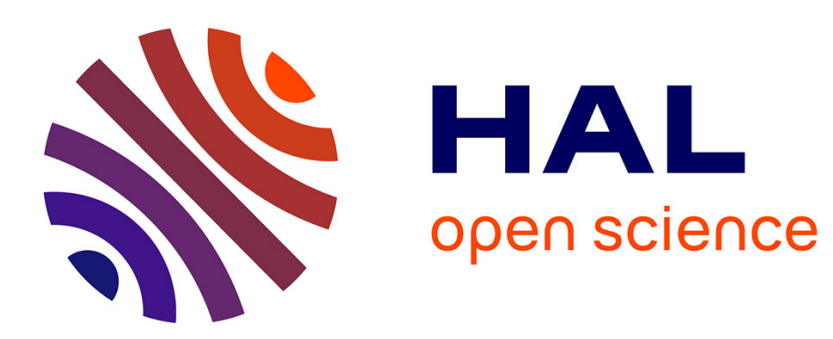

\title{
Modalité d'interaction humaine dans la formation en ligne: son influence sur l'apprentissage
}

Elke Nissen

\section{To cite this version:}

Elke Nissen. Modalité d'interaction humaine dans la formation en ligne: son influence sur l'apprentissage. Savoirs: Revue internationale de recherches en éducation et formation des adultes, 2005, Analyse de l'activité et formation, 8, pp.89-106. edutice-00001447

HAL Id: edutice-00001447

https://edutice.archives-ouvertes.fr/edutice-00001447

Submitted on 12 Apr 2006

HAL is a multi-disciplinary open access archive for the deposit and dissemination of scientific research documents, whether they are published or not. The documents may come from teaching and research institutions in France or abroad, or from public or private research centers.
L'archive ouverte pluridisciplinaire HAL, est destinée au dépôt et à la diffusion de documents scientifiques de niveau recherche, publiés ou non, émanant des établissements d'enseignement et de recherche français ou étrangers, des laboratoires publics ou privés. 
Elke Nissen, Modalité d'interaction humaine dans la formation en ligne : son influence sur l'apprentissage, Revue Savoirs « Les TIC à l'usage pour la formation » numéro 8/2005, 89-106.

\title{
Modalité d'interaction humaine dans la formation en ligne : son influence sur l'apprentissage
}

Résumé

La présence de l'interaction humaine dans une formation en ligne est s'accrue avec l'évolution des technologies de la communication et fait partie aujourd'hui des composantes habituelles de ces formations. L'interaction sociale est vue comme un facteur d'apprentissage. Or, la question se pose de savoir quelle modalité d'interaction est la plus adaptée pour un type de tâche et pour un contexte donnés. A côté d'un tutorat qui paraît indispensable pour l'orientation des étudiants à distance, le groupe restreint apporte-t-il des bénéfices ? Les modèles théoriques généralement utilisés sont issus de l'enseignement présentiel et ne mettent pas en parallèle une situation d'interaction en groupe tutoré et une autre en individuel tutoré. Notre recherche empirique a ainsi pour objectif de voir laquelle des deux modalités d'interaction est plus adaptée dans une formation en langues à distance qui se situe dans une perspective actionnelle. Elle compare les deux modalités, et cela successivement sur deux plate-formes. Le groupe a tendance à progresser davantage que les individuels tutorés dans la qualité de ses rédactions durant la phase de formation. En revanche, en termes d'apprentissage, aucune différence significative entre les deux populations ne peut être observée. Les phénomènes observés restent stables d'une plate-forme à l'autre, faisant ainsi apparaître le groupe restreint tutoré, dans le contexte donné, comme un moyen performant pour produire, mais pas forcément pour apprendre.

Mots-clés

Modalité d'interaction humaine, apprentissage collaboratif, formation à distance, perspective actionnelle en langues

\section{Human interaction in an e-learning set-up : its influence on acquisition}

\begin{abstract}
With the evolution of communication technology, human interaction becomes more and more present in e-learning environments. It is considered benefiting acquisition. But what mode of interaction is the most adequate in a given context and for a given task ? Tutoring seems to be indispensable to the learners' orientation at a distance. But, does a tutored group of peers benefit more to the learner than individual tutoring without group interaction does ? The theories of interaction that are generally evoked do not compare these two modes of tutored interaction. But this exactly is the purpose of our empirical research : it aims to find out which mode of interaction benefits more to the learner in an action-oriented language e-learning set-up. The experiment was conducted successively on two learning platforms. The results of the study show no significant difference between the acquisition of the students who worked in a tutored group and those who were tutored individually. But, groups tend to increase the quality of task accomplishment during the training period. This phenomenon occurs on both platforms. Thus, in the given context, small tutored groups help the learners to accomplish the task better, but not to learn better.
\end{abstract}

Key-words

Human interaction, collaborative learning, e-learning, action-oriented approach in language learning 


\section{Introduction : Introduction de l'interaction humaine dans la formation à distance}

Depuis ses débuts, l'évolution de la formation à distance (FAD) est liée de près au développement des systèmes et outils techniques qui y sont mis à profit. De la même manière, l'interaction humaine dans le cadre de la FAD s'est renforcée avec le développement des outils numériques. Ainsi, lorsque la naissance du timbre a permis l'apparition de la formation par correspondance au milieu du $19^{\mathrm{e}}$ siècle, le contact entre enseignant et apprenant par courrier restait relativement rare et espacé dans le temps; la formation consistant essentiellement en l'envoi de cours ou de manuels à l'apprenant. A partir de la fin des années 1980 sont introduits dans la FAD d'autres outils de communication : le téléphone et le fax, qui servent à la communication entre l'apprenant et un tuteur. La communication entre apprenants naît essentiellement avec le développement des réseaux locaux (Local Area Networks ou LAN), mais les personnes se trouvent encore dans un même lieu, voire une même salle d'ordinateurs. Les réseaux locaux sont au milieu des années 1990 remplacés par le réseau mondial internet. L'apparition et le développement des outils de communication numériques, qui utilisent le réseau internet - tels que le courriel, le chat, le forum de discussion, l'audioconférence, la visioconférence, etc. - rendent possible une interaction plus rapide et plus fréquente entre le tuteur-enseignant et l'apprenant ou bien entre les différents apprenants. Aujourd'hui, surtout dans ce qui est appelé les «campus numériques », le média utilisé est souvent exclusivement internet et l'interaction qu'il véhicule est le plus souvent écrite. Si l'utilisation du multimédia dans la FAD y avait déjà introduit de l'interactivité, c'est-à-dire un dialogue entre homme et machine (Demaizière \& Dubuisson 1992 : 28) dans lequel l'ordinateur réagit aux actions de l'homme en ouvrant une page-écran ou en donnant un feed-back préprogrammé par exemple, la généralisation d'internet offre non seulement accès à des informations et ressources au niveau mondial, mais permet aussi l'interaction interpersonnelle à travers le monde (Chapelle $2001: 20$ 26). L'apprenant peut, de plus en plus facilement et en profitant des possibilités d'interaction humaine que les technologies offrent, interagir avec un tuteur ou en groupe avec des pairs dans le cadre de sa formation. Dans les campus numériques, il existe d'ailleurs une forte tendance de travail « collaboratif » en groupe restreint.

Or, dans un cadre d'apprentissage d'une langue étrangère donné, quelle modalité d'interaction favorise le mieux l'apprentissage à distance ? Le tutorat, qui a préalablement été pratiqué dans la FAD et qui est régulièrement éprouvé par la pratique dans des centres d'autoformation ? Ou bien 
l'apprentissage en groupe de pairs, très en vogue dans la FAD ? Les recherches qui ont pour objectif de répondre à cette question pour la FAD restent rares, tout d'ailleurs comme pour l'enseignement présentiel. La modalité du tutorat individuel et celle de l'apprentissage en groupe (tutoré ou non) ne sont à notre connaissance jamais comparées expérimentalement entre elles, mais seulement séparément l'une ou l'autre avec un apprenant travaillant totalement seul.

\section{Quel type d'interaction est le plus adapté dans une FAD ?}

Le contexte dans lequel se situe notre recherche est celui d'une formation en langues à distance. Le(s) dispositif(s) de formation concerné(s) étaient en cours d'élaboration au moment de la recherche, qui a eu ainsi pour objectif de répondre à une question pédagogique réelle : quelle modalité interactionnelle proposer aux apprenants à distance? Nous exposerons d'abord la démarche didactique inhérente aux formations en question, puis les modèles théoriques qui mettent en avant les bénéfices de l'une ou de l'autre modalité interactionnelle, avant de formuler notre hypothèse de recherche.

\section{II.1. Une FAD en langues}

\section{L'approche didactique retenue}

L'approche didactique en vigueur dans les dispositifs de formation en question est la perspective actionnelle. Le Conseil de l'Europe introduit cette perspective avec le Cadre européen commun de référence pour les langues (2001; voir aussi l'édition de 1998), sans pourtant la distinguer clairement de l'approche communicative, qui est à ce jour la méthodologie la plus répandue dans l'enseignement et l'apprentissage des langues en Europe. Cette publication amorce ainsi des changements que les dernières publications de Puren (par exemple Puren 2002a, 2002b) permettent de comprendre plus aisément.

Dans une Europe dans laquelle les contacts entre ses citoyens deviennent de plus en plus quotidiens et habituels, que ce soit lors d'échanges universitaires ou dans l'entreprise par exemple, l'apprenant visé n'est plus le touriste en contact occasionnel avec le locuteur étranger, mais celui qui est régulièrement en contact avec la langue cible. Tandis que dans l'approche communicative, la communication est quelquefois considérée comme une fin en soi et se cherche un but authentique (Poteaux 1998), l'interaction sociale sert dans la perspective actionnelle à la réalisation d'une tâche en langue cible (ou parfois non langagière, comme le montage d'une tente). Cette tâche relève d'un 
contexte plausible pour l'apprenant, c'est-à-dire d'un contexte de travail, de formation ou du quotidien. L'apprenant - en tant qu'acteur social - interagit en langue étrangère afin d'accomplir la tâche. Les compétences nécessaires se situent ainsi à plusieurs niveaux : au niveau langagier, au niveau de l'interaction humaine, mais également au niveau de la réalisation de la tâche.

L'objectif d'apprentissage visé dans le dispositif de formation en question est celui de la réalisation d'une tâche en langue étrangère. Par exemple dans CLES (la certification de compétences en langues de l'enseignement supérieur), une certification qui va être introduite prochainement dans les universités, écoles supérieures et instituts de formation des maîtres en France (Ministère de l'Éducation Nationale 2001), l'évaluation des apprenants reste essentiellement individuelle. Cette certification s'inscrit dans ses grandes lignes dans la perspective actionnelle, mais fait appel à des compétences individuelles de compréhension et de rédaction; seul un échange de type questionsréponses après une présentation orale de l'étudiant y est prévu au bout de la première phase expérimentale de la certification. Un des objectifs principaux du premier dispositif de formation à distance dont il est question ici est la préparation des étudiants à CLES. Les compétences visées dans le dispositif sont donc individuelles. Néanmoins, l'interaction sociale est considérée, au vu des modèles théoriques exposés ci-dessous, comme une aide potentielle pour l'apprenant dans sa construction individuelle des connaissances et compétences; présupposé que nous cherchons justement à mettre à l'épreuve à travers l'expérimentation décrite plus loin. Notre cas de figure est très proche de celui d'autres dispositifs de formation à distance, et notamment ceux qui préconisent le travail en groupe: les formations conjointes entre pairs, passant par la réalisation en groupe tutoré de différentes tâches dans des situations d'apprentissage, sont régulièrement pourvues d'une évaluation finale individuelle. C'est le cas par exemple des formations dispensées sur Acolad, la plate-forme qui est utilisée pour notre deuxième dispositif de formation. Par ailleurs, les études conduites pour montrer l'efficacité du groupe pour l'apprentissage, en présentiel - qui sont à la base des théories sur l'apport du conflit socio-cognitif (voir ci-dessous) par rapport à un apprentissage sans contact social utilisent un schéma expérimental qui évalue les apprenants de manière individuelle et non collective (Gilly 1995).

\section{Les dispositifs de FAD en langues observés}

Le premier dispositif de formation en langues dont il est question ici s'inscrit dans le contexte universitaire. Il est conçu grosso modo comme un centre de ressources en langues en ligne. Il 
propose un ensemble de ressources (textuelles, audio, vidéo) en langue étrangère, de liens vers des sites internet intéressants, des aides langagières, ainsi que des aides méthodologiques avec des exercices d'application interactifs. L'apprenant met à profit ces aides dans la réalisation de tâches de production de type universitaire, du même type que celles qui peuvent être demandées dans le cadre de CLES, telles qu'un résumé, un exposé, le compte-rendu d'un article scientifique ou une synthèse par exemple. Le choix pédagogique a été fait de proposer systématiquement un tutorat à l'étudiant, que cet accompagnement soit complété par une interaction en groupe restreint ou non. Le tuteur a pour fonction de guider l'apprenant dans le choix de ses activités, de faciliter son apprentissage, de répondre aux éventuelles questions, de donner des retours sur la langue et sur le contenu par rapport aux productions intermédiaires ou finales que lui remet l'étudiant. De plus, lorsqu'il s'agit d'une activité en groupe, le tuteur aide le groupe à se gérer et à organiser le fonctionnement commun. Toutes les interactions se font à distance, par le biais d'outils de communication numériques, et en langue étrangère - à moins que l'apprenant ne sache absolument pas comment exprimer une chose en langue cible; dans ce cas il a recours à la langue maternelle, mais ce cas de figure est resté extrêmement rare pendant la phase d'expérimentation (Nissen 2003 : 114). Les étudiants qui ont choisi la modalité du petit groupe tutoré (groupes allant jusqu'à quatre personnes) préparent ensemble une rédaction. Ils prennent connaissance des documents proposés sur la plate-forme dédiée à la formation ou alors en cherchent eux-mêmes sur internet. Ils discutent via un chat, le courriel et un forum spécifique sur les idées centrales de ces documents, puis, à partir de ces idées, négocient la structure à retenir pour leur rédaction. Chaque membre du groupe rédige ensuite une partie ; à la fin, chacun relit l'ensemble du texte en veillant à le rendre homogène et en proposant des modifications et des corrections. Le tuteur n'intervient dans la mesure du possible pas pendant les phases de négociation au sein du groupe, afin de favoriser la discussion entre pairs (ils ont tendance sinon à trop se concentrer sur la «parole d'expert» du tuteur). Il intervient essentiellement suite aux propositions communes du groupe, après chacune des phases successives dans l'accomplissement de la tâche de rédaction.

Ce dispositif, Babbelnet, a été conçu par le service pédagogique interuniversitaire de ressources pour l'autoformation en langues (SPIRAL) de Strasbourg et a été proposé, en allemand et en anglais, aux étudiants des trois universités strasbourgeoises. Les étudiants provenaient ainsi de filières diverses. Leur participation a eu lieu soit en dehors de leur cursus, soit y a été validée par une note après accord de l'enseignant de langue de leurs cursus respectifs. Cette offre a été 
complétée par un enseignement d'ouverture en allemand (c'est-à-dire un enseignement qui s'ajoute à la filière principale des étudiants volontaires et dont la note finale compte pour leur année universitaire si elle est au-dessus de la moyenne), proposé aux étudiants de première et deuxième années des filières scientifiques. L'expérimentation a porté sur la réalisation d'une synthèse en allemand, avec l'aide d'un tuteur et éventuellement d'un groupe de pairs, à partir d'un corpus de documents sur une thématique commune et d'actualité sociale. 23 étudiants ont participé à la première expérimentation, dont 15 en modalité de groupe et 8 en modalité individuelle (les étudiants dans les deux modalités étant accompagnés par un tuteur). Le détails de ce programme expérimental est exposé dans une article séparé (Nissen 2004).

Le deuxième dispositif d'observation, qui a pour fonction de compléter le premier et de vérifier la validité des résultats obtenus sur une plate-forme avec des fonctionnalités techniques différentes et dans une autre langue, a été réalisé non sur la plate-forme conçue pour Babbelnet, mais sur Acolad, une plate-forme élaborée par l'université Strasbourg 1. La tâche proposée (la synthèse) et les aides méthodologiques, les types d'aides langagières et de ressources, ainsi que le mode d'intervention du tuteur restent identiques par rapport à la première expérimentation, hormis le fait qu'ils sont cette fois en anglais. La formation dans laquelle s'inscrit le module d'anglais mis en place selon ces conditions et qui est observé pour les fins de notre étude est une licence professionnelle en ligne portant sur les «activités et techniques de communication». 16 étudiants ont participé à cette deuxième expérimentation, dont 11 en modalité de groupe et 5 en modalité individuelle. Le choix de la modalité interactionnelle a été effectué par les étudiants eux-mêmes.

\section{II.2. Modèles théoriques des modalités d'interaction}

L'apport d'une relation interpersonnelle dans l'avancement cognitif personnel est admis dans des types de situations différentes, où l'apprenant interagit avec une personne plus expérimentée ou avec des pairs; en formation de langues, elle est vue comme un facteur primordial de progrès ( $c f$. Warschauer 1998). De même que les études expérimentales comparant l'apprentissage à travers les différentes modalités d'interaction font défaut, les modèles théoriques disponibles sont formulés soit en faveur de l'un, soit de l'autre type d'interaction, sans mettre en relation les deux.

\section{Apprendre avec un tuteur :}


Vygotsky (1985) est un des premiers à avoir mis en avant la nature sociale de l'apprentissage. Selon lui, les processus mentaux supérieurs de l'enfant se développent plus facilement à travers un contact social favorable. Dans son approche socio-constructiviste, le contexte social est un élément constitutif de l'apprentissage, et par là du développement, dans la relation entre l'individu et l'objet (la tâche). C'est d'une relation dissymétrique, entre un novice et un autre individu plus expert, que parle Vygotsky. Bruner (1983), un des successeurs de Vygotsky, met surtout en avant l'imitation comme processus d'apprentissage dans la situation d'interaction dissymétrique. L'expert, ou le tuteur, joue le rôle de modèle, que l'apprenant peut imiter lorsque son niveau initial de développement le lui permet : il arrive ainsi à faire certaines choses qu'il ne saurait pas faire en situation autonome. Cette imitation n'est pas un simple mimétisme, mais un apprentissage par observation. Par ailleurs, le tuteur remplit un rôle d'étayage; il veille par exemple à ce que l'apprenant ne dévie pas de l'objectif fixé ou l'aide à trouver les traits essentiels de la tâche afin qu'il trouve plus facilement une réponse adéquate. Ces auteurs mettent ainsi en avant la facilitation de l'apprentissage par un tuteur (ou un être plus avancé). Ces fondements théoriques ont été élaborés à partir d'observations et d'expérimentations dans des situations présentielles. Dans la $\mathrm{FAD}$, sans doute à cause du coût élevé d'un tutorat individuel, ils ne sont généralement pas mis à l'épreuve.

\section{Apprendre en groupe restreint :}

La pratique de plus en plus répandue de l'apprentissage collaboratif en FAD, en revanche, est généralement justifiée par la référence à Vygotsky et aux travaux de l'École de Genève sur les apports du conflit socio-cognitif. Doise, Mugny et Perret-Clermont (par exemple, Doise \& Mugny 1981, Perret-Clermont 1996) notamment montrent que, dans une dyade symétrique entre pairs, le fait de surmonter un conflit entre leurs manières de répondre à un problème est particulièrement propice au développement cognitif des individus impliqués. Or, de nombreuses conditions doivent être réunies pour que le conflit socio-cognitif se produise et soit bénéfique. Tout d'abord, une contradiction entre les solutions proposées par les apprenants doit se produire. Cela doit amener l'individu à remettre en question sa réponse et il doit accepter de participer activement à la recherche d'une solution commune; cette recherche se situe obligatoirement au niveau intellectuel et non relationnel pour être efficace. Vu le nombre de contraintes, Gilly indique que le conflit sociocognitif se produit en réalité rarement. En revanche, la co-élaboration acquiéscante, lors de laquelle 
un individu propose et l'autre approuve simplement, peut également être bénéfique. Il en est de même pour la confrontation avec désaccord, dans laquelle un individu récuse la solution de l'autre sans argumenter (Gilly 2001 : 27). L'imitation et l'étayage, quant à eux, ne se produisent pas exclusivement en situation d'interaction dissymétrique mais peuvent également avoir lieu en dyade symétrique (Rémigy 2001). Ainsi, d'autres éléments que le conflit socio-cognitif justifient également l'apprentissage collectif.

De nombreux auteurs distinguent deux types d'apprentissage collectif : l'apprentissage coopératif et l'apprentissage collaboratif (par exemple, Panitz 1997, Henri \& Lundgren-Cayrol 2001). Si les deux sont basés sur une approche socio-constructiviste de l'apprentissage, dans la coopération, l'accent est davantage mis sur le produit final. Les apprenants y procèdent fréquemment par répartition des tâches, puis par un assemblage final. Le tuteur y joue un rôle relativement important. Dans la coopération, l'investissement de l'apprenant dans le groupe est plus grand et le rôle du tuteur du même coup réduit. Ce sont l'apprentissage et l'entre-aide qui sont placés au centre de la démarche ; ce qui suppose que chaque membre du groupe ait la volonté de participer à la construction de connaissances des autres. En réalité, la coopération et la collaboration cohabitent la plupart du temps et sont mises en œuvre à différents moments de l'accomplissement de la tâche par le groupe. Nous désignerons les deux d'apprentissage collectif ou bien d'apprentissage en petit groupe tutoré.

\section{II.3. Hypothèse}

La confrontation des modèles théoriques en faveur de la modalité d'interaction entre un apprenant et un tuteur $v s$. entre apprenants ne permet a priori pas de conclure sur la supériorité d'une modalité d'interaction par rapport à l'autre. Par contre, dans notre cas, les choix pédagogiques retenus sont ceux d'un accompagnement individuel par le tuteur ou bien d'une interaction en groupe tutoré ; un travail à distance sans tuteur étant considéré comme trop déstabilisant pour les apprenants qui demandent dans les FAD d'avoir la possibilité de savoir s'ils s'orientent dans la bonne direction. La présence d'un tuteur permet également de s'assurer que l'interaction se déroule autant que possible en langue étrangère. L'intervention du tuteur est ainsi prévue également dans la situation d'interaction symétrique, sans que des modèles théoriques pour ce type de situation d'apprentissage soient disponibles.

Dans l'élaboration de notre hypothèse de recherche, deux arguments s'affrontent. D'une part, bien que dans le cas du groupe tutoré, l'accompagnement du tuteur s'ajoute à la co-élaboration entre 
pairs, ses interventions ne s'adressent pas aux individus qui constituent le groupe mais seulement au groupe en entier. Les réactions, conseils ou corrections ne sont par conséquent pas ciblés spécifiquement pour un apprenant, contrairement au mode individuel tutoré. Pour un apprentissage individuel, des conseils ciblés peuvent cependant représenter un atout notable, comme l'ont montré les travaux de Bruner. D'autre part, l'interaction en groupe intervient au moment-même où l'individu se pose la question de savoir comment répondre au mieux au problème posé ; les travaux sur le conflit socio-cognitif ont mis en avant l'importance du moment (c'est-à-dire lorsque l'apprenant en a besoin) des remarques ou critiques pour l'apprentissage. Le tuteur, en revanche, n'intervient sur le contenu qu'à des moments prédéfinis, une fois que les apprenants ont proposé leur solution au problème. De plus, en situation d'interaction symétrique, l'apprenant peut discuter les commentaires avec ses pairs ou les critiquer lui-même, vu qu'il n'a pas de rapport hiérarchique avec eux - contrairement au rapport avec le tuteur. Nous supposons que cela l'aide à mettre en œuvre et à développer davantage ses compétences métacognitives (conformément à Linard 2003). De plus, en groupe tutoré l'apprenant n'échange pas seulement avec le tuteur mais également avec les pairs ; il pratique ainsi davantage la langue étrangère que la personne en mode individuel tutoré. Pour ces raisons, nous nous attendons à une supériorité de la modalité du groupe tutoré par rapport à la modalité individuelle tutorée.

\section{Observations et discussion}

Afin de savoir quel mode d'interaction, en individuel tutoré ou en groupe restreint tutoré, est plus adapté à notre dispositif de formation en ligne pour les langues étrangères qui s'inscrit dans la perspective actionnelle, nous comparons les résultats des apprenants ayant suivi une formation selon l'une ou l'autre modalité interactionnelle. La phase de formation des apprenants a consisté en la réalisation successive de deux synthèses, selon la modalité choisie. En amont, ils ont rédigé une synthèse à partir d'un ensemble de documents en langue étrangère et avec l'aide d'une fiche méthodologique exposant le type de résultat attendu et d'un dictionnaire. Après la phase d'apprentissage, ils ont rédigé une dernière synthèse, dans des conditions identiques que lors du prétest. Afin d'assurer une plus grande objectivité dans l'évaluation, les rédactions ont été évaluées par deux correcteurs, selon une même grille de critères (portant sur la qualité langagière ainsi que sur la qualité de l'argumentation et de la structuration de la synthèse). C'est la moyenne des scores attribués par les deux correcteurs qui a été prise en compte. La difficulté pour l'élaboration des 
épreuves ainsi que de la grille d'évaluation a été de se rapprocher le plus possible de CLES, puisque c'est la préparation à cette certification qui nous avait amenée à nous poser la question de la modalité d'interaction la plus adaptée, sans cependant disposer d'un descriptif exhaustif et définitif de la certification. En effet, la conception définitive de cette dernière, ainsi que sa mise en place, devaient initialement être terminées au début de notre expérimentation (en automne 2002), mais n'étaient finalement pas encore disponibles.

\section{III.1. Aucune influence de la modalité d'interaction sur l'apprentissage n'est visible}

Les résultats des deux populations, l'une ayant suivi la formation en mode individuel tutoré et l'autre en groupe tutoré, ont été analysés au moyen de tests statistiques ${ }^{1}$. Le score pour chaque synthèse tient compte de trois aspects, qui sont l'aspect pragmatique, l'aspect cognitif et l'aspect langagier.

- L'aspect pragmatique concerne l'usage effectif de la langue étrangère dans l'accomplissement d'une tâche en langue étrangère, c'est-à-dire l'organisation du discours, la manière dont la synthèse présente la problématique commune aux documents de départ, y compris dans l'introduction et la conclusion.

- L'aspect cognitif, quant à lui, porte sur la capacité de l'apprenant à identifier les idées essentielles dans les documents fournis et de les présenter dans la synthèse.

- L'aspect langagier correspond aux compétences de l'expression écrite : à la reformulation des informations contenues dans les documents, à l'emploi d'un lexique varié et maîtrisé, à des phrases syntaxiquement et morphologiquement correctes et adaptées, ainsi qu'à une bonne orthographe.

L'analyse des productions écrites des apprenants au début et à la fin du premier dispositif pédagogique montre que les deux populations s'améliorent pour l'aspect pragmatique, mais sans qu'une population s'améliorerait plus que l'autre (le tableau 1 indique les détails des différentes analyses statistiques). Pour l'aspect langagier, les personnes travaillant en mode individuel tutoré et celles travaillant en groupe tutoré n'évoluent pas non plus différemment. Pour deux critères

\footnotetext{
${ }^{1}$ Ils ont été analysés grâce à un test non paramétrique (Kruskal-Wallis), ainsi qu'un test paramétrique (ANOVA) afin de nous assurer que les conditions pour l'application d'un test statistique paramétrique pour la comparaison entre le pré- et le post-test étaient remplies. Lorsque les deux tests statistiques montraient une différence de significativité, nous n'avons pas poursuivi les analyses pour la variable en question. Dans le cas contraire, le test de l'analyse de la variance (ANOVA) à mesures répétées a été utilisé quand la différence entre les populations n'a pas été significative pour le prétest. Sinon, lorsque les populations avaient manifesté un niveau significativement différent au prétest, c'est le test de la covariance (ANCOVA) qui a été employé pour comparer l'évolution des populations entre le prétest et le post-test.
} 
d'analyse (la totalité du score obtenu pour chaque rédaction et l'aspect cognitif), les conditions d'application du test de la covariance ne sont pas remplies. Nous ne sommes par conséquent pas en mesure de nous prononcer sur la significativité des différences entre les populations concernant ces aspects.

Dans le deuxième dispositif de formation, l'analyse de la totalité du score montre que la différence les deux populations n'évoluent pas différemment l'une de l'autre entre le pré- et le post-test. Il apparaît d'ailleurs qu'elles ne progressent pas significativement ni l'une, ni l'autre. Lorsqu'on regarde de plus près les différents critères retenus pour l'analyse des résultats, les mêmes tendances se dessinent. En effet, ni pour l'aspect pragmatique, ni pour l'aspect langagier, une différence de progrès entre les deux populations ou bien une amélioration significative ne s'observe. Pour l'aspect cognitif, les conditions d'applicabilité des tests statistiques paramétriques n'étaient pas remplies et nous ne pouvons ainsi pas tirer de conclusions sur l'évolution de cet aspect.

\begin{tabular}{|c|c|c|c|c|c|c|c|c|c|}
\hline & & \multicolumn{4}{|c|}{ Dispositif pédagogique 1} & \multicolumn{4}{|c|}{ Dispositif pédagogique 2} \\
\hline & & prag2. & $\operatorname{cog}^{3}$ & lang ${ }^{2}$. & score total $^{2}$ & prag $^{2}$. & $\operatorname{cog}$ & lang ${ }^{2}$. & score total ${ }^{2}$ \\
\hline $\begin{array}{l}\text { Prétest / } \\
\text { post-test }\end{array}$ & $\begin{array}{l}\text { T: } \\
\text { I : }\end{array}$ & $\begin{array}{l}\mathrm{F}=32.341 ; \\
\mathrm{p}<.0001 \\
\mathrm{~F}=1.614 ; \\
\mathrm{ns} .\end{array}$ & $\begin{array}{l}\text { ANCOVA } \\
\text { pas } \\
\text { possible }\end{array}$ & $\begin{array}{l}\mathrm{F}=2.574 ; \\
\text { ns. }\end{array}$ & $\begin{array}{l}\text { ANCOVA } \\
\text { pas } \\
\text { possible }\end{array}$ & $\begin{array}{l}\mathrm{F}=1.167 ; \\
\text { ns. } \\
\mathrm{F}=0.214 ; \\
\text { ns. }\end{array}$ & $\begin{array}{l}\text { ANOVA } \\
\text { pas } \\
\text { possible }\end{array}$ & $\begin{array}{l}\mathrm{F}=2.279 ; \\
\text { ns. } \\
\mathrm{F}=0.349 ; \\
\text { ns. }\end{array}$ & $\begin{array}{l}\mathrm{F}=1.743 ; \\
\mathrm{ns} . \\
\mathrm{F}=0.328 ; \\
\text { ns. }\end{array}$ \\
\hline
\end{tabular}

Tableau 1. Effets du temps (T) et effets du mode d'interaction (I) sur les aspects pragmatique (prag.), cognitif (cog.) et langagier (lang.) et le score total des rédactions entre le prétest le posttest.

Pour résumer, l'analyse des scores obtenus pour le pré- et le post-test ne fait globalement pas apparaître de différence significative entre les progrès des deux populations ayant profité d'un mode d'interaction différent (individuel vs. groupe). Cela s'observe invariablement dans les deux dispositifs de formation. La modalité d'interaction sociale ne semble ainsi pas influencer l'apprentissage individuel dans le contexte décrit de cette formation d'une durée moyenne (environ trois mois) à distance.

\section{III.2. Le groupe tutoré est plus performant que les individus}

Nous venons de comparer les résultats au post-test à ceux du prétest afin de savoir si une population avait amélioré plus que l'autre sa réalisation individuelle du type de tâche retenu. Nous allons maintenant regarder de près la performance des populations durant la formation. Le tableau 2

\footnotetext{
${ }^{2}$ Ces résultats ont été obtenus à l'aide du test de la variance à mesure répétée.
} 
montre les résultats des tests statistiques pour la comparaison des scores obtenus par les deux populations à différents moments de la formation. Ainsi, nous comparons les scores obtenus

- lors du pré-test (en situation de test individuelle) et lors de la première synthèse rédigée pendant la phase de formation (soit en individuel tutoré, soit en groupe tutoré) (S1),

- pour la première et la seconde synthèse de la phase d'apprentissage (S1, S2), toujours selon la même modalité d'interaction (individuelle tutorée $v s$. en groupe restreint tutoré), et

- pour la deuxième synthèse réalisée durant la phase de formation (S2) (en individuel tutoré $v s$. en groupe tutoré) et celle du post-test (rédigée en situation de test individuelle).

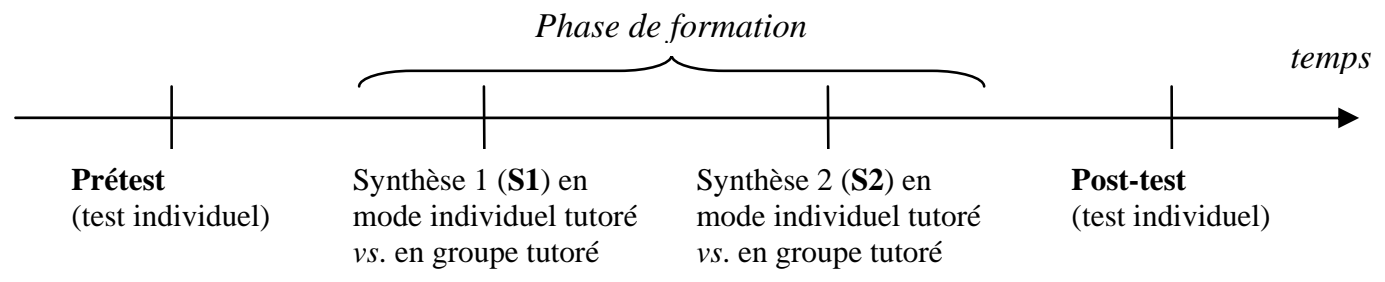

Figure 1. Aperçu des quatre moments d'évaluation

Dans le premier dispositif pédagogique, lorsque les apprenants passent d'une situation de test individuelle (le prétest) à une rédaction réalisée avec l'aide d'un tuteur et éventuellement d'un groupe de pairs (S1), leurs scores restent stables. Les deux populations (individuels tutorés $v s$. petits groupes tutorés) n'évoluent pas différemment. Par contre, des évolutions sont ensuite visibles au cours des rédactions réalisées pendant la phase de formation (S1 et S2) : Les étudiants baissent leur performance pragmatique. Les commentaires faits par les apprenants après l'expérimentation nous amènent à attribuer cette baisse de niveau d'une part à une plus grande difficulté du sujet de la seconde synthèse (S2), et d'autre part à une lassitude des étudiants à chercher une structure claire et cohérente aux idées identifiées dans les documents de départ; ils ont maintenant l'impression de « savoir déjà faire ». Pour ce même aspect pragmatique, les deux populations évoluent de manière différente: les groupes gardent un niveau plus constant que les personnes travaillant en mode individuel tutoré, dont la performance baisse davantage. Puis, entre la seconde synthèse (S2) et le post-test, les deux populations améliorent la qualité pragmatique de leurs rédactions (qui était particulièrement basse dans la seconde synthèse), mais en revanche baissent leur performance langagière écrite. Rappelons qu'aucune différence significative n'existe entre le pré- et le post-test.

\footnotetext{
${ }^{3}$ Ces résultats ont été obtenus à l'aide du test de la covariance (ANCOVA).
} 
Dans le deuxième dispositif pédagogique, la performance des étudiants s'améliore dès qu'ils passent d'une condition de test (le prétest) à un mode interactionnel pendant la phase de formation. Ainsi, les deux populations s'améliorent entre le prétest individuel et la première synthèse rédigée durant la formation (S1), qu'ils l'aient réalisée individuellement avec un tuteur ou bien en groupe tutoré. De la première à la seconde synthèse de la période de formation ( $\mathrm{S} 1$ à S2), le niveau pragmatique des rédactions baisse tout comme dans le premier dispositif observé, et ce pour les deux populations. Pour le niveau langagier, une divergence apparaît entre les deux populations. Les individus tutorés baissent leur niveau, tandis que les groupes tutorés améliorent le leur. Puis, de la seconde synthèse de la phase de formation au post-test, l'on observe une baisse de niveau, tout comme cela était le cas dans le premier dispositif pédagogique. En effet, cette fois, aussi bien la qualité cognitive que langagière des rédactions diminuent. Pour l'aspect langagier, les personnes ayant profité de la modalité d'interaction en groupe tutoré baissent davantage leur niveau que ceux ayant travaillé en individuel tutoré.

De manière globale, il apparaît ainsi que la qualité des synthèses a tendance à être meilleure en situation d'interaction sociale, lors de la phase de formation, que lors des tests où aucune interaction n'est possible. Les étudiants apportent cependant moins de soin à la structuration cohérente de leur synthèse lorsque l'expérience se répète (c'est-à-dire pour la seconde synthèse en phase de formation) ; ce phénomène est d'autant plus vrai quand les étudiants sont en interaction seulement avec un tuteur et non avec un groupe. Lorsque des différences entre les populations sont visibles, elles suggèrent toujours que le groupe s'améliore au fur et à mesure qu'il travaille (c'est-à-dire de la première à la seconde synthèse durant la formation), et progresse ainsi davantage que les individuels. Rappelons que les personnes en modalité d'interaction du groupe tutoré bénéficient à la fois de la confrontation des avis entre pairs et de l'accompagnement d'un tuteur.

\begin{tabular}{|c|c|c|c|c|c|c|c|}
\hline & & \multicolumn{3}{|c|}{ Dispositif pédagogique 1} & \multicolumn{3}{|c|}{ Dispositif pédagogique 2} \\
\hline & & prag 4. & $\operatorname{cog}^{5}$ & lang 5. & prag $^{5}$. & $\operatorname{cog}$. & lang 5. \\
\hline Prétest / S1 & $\begin{array}{l}\text { T: } \\
\text { I : }\end{array}$ & $\begin{array}{l}\mathrm{F}=1.337 ; \mathrm{ns} \\
\mathrm{F}=2.068 ; \mathrm{ns}\end{array}$ & $\begin{array}{l}\text { ANCOVA pas } \\
\text { possible }\end{array}$ & $\begin{array}{l}\mathrm{F}=2.195 ; \mathrm{ns} \\
\mathrm{F}=0.367 ; \mathrm{ns} .\end{array}$ & $\begin{array}{l}\mathrm{F}=9.320 ; \mathrm{p}=.01 \\
\mathrm{~F}=1.154 ; \mathrm{ns} .\end{array}$ & $\begin{array}{l}\text { ANCOVA pas } \\
\text { possible }\end{array}$ & $\begin{array}{l}\mathrm{F}=6.603 ; \mathrm{p}<.05 \\
\mathrm{~F}=0.033 ; \mathrm{ns} .\end{array}$ \\
\hline S1 / S2 & $\begin{array}{l}\text { T: } \\
\text { I : }\end{array}$ & $\begin{array}{l}F=4.064 ; p<.10 \\
F=3.440 ; p<.10\end{array}$ & $\begin{array}{l}\text { ANCOVA pas } \\
\text { possible }\end{array}$ & $\begin{array}{l}\mathrm{F}=0.238 ; \mathrm{ns} \\
\mathrm{F}=0.001 ; \mathrm{ns} .\end{array}$ & $\begin{array}{l}F=4.509 ; p \leq .05 \\
F=0.109 ; n s .\end{array}$ & $\begin{array}{l}\text { ANCOVA pas } \\
\text { possible }\end{array}$ & $\begin{array}{l}\mathrm{F}=1.885 ; \mathrm{ns} . \\
\mathrm{F}=44.935 ; \\
\mathrm{p}<.0001\end{array}$ \\
\hline S2 / post-test & $\begin{array}{l}\text { T: } \\
\text { I : }\end{array}$ & $\begin{array}{l}\mathrm{F}=5.031 ; \mathrm{p}<.05 \\
\mathrm{~F}=0.157 ; \mathrm{ns} .\end{array}$ & $\begin{array}{l}\mathrm{F}=0.605 ; \mathrm{ns} . \\
\mathrm{F}=0.605 ; \mathrm{ns} .\end{array}$ & $\begin{array}{l}\mathrm{F}=5.524 ; \mathrm{p}<.05 \\
\mathrm{~F}=4.197 ; \mathrm{ns} .\end{array}$ & $\begin{array}{l}\mathrm{F}=0.913 ; \mathrm{ns} . \\
\mathrm{F}=1.476 ; \mathrm{ns} .\end{array}$ & $\begin{array}{l}\mathrm{F}=7.253 ; \mathrm{p}<.05 \\
\mathrm{~F}=0.114 ; \text { ns. }^{3}\end{array}$ & $\begin{array}{l}F=5.903 ; p<.05 \\
F=5.400 ; p<.05\end{array}$ \\
\hline
\end{tabular}

\footnotetext{
${ }^{4}$ Ces résultats ont été obtenus à l'aide du test de la variance à mesure répétée.

${ }^{5}$ Ces résultats ont été obtenus à l'aide du test de la covariance (ANCOVA).
} 
Tableau 2. Effets du temps (T) et effets du mode d'interaction (I) d'une synthèse à l'autre (S1, S2 = respectivement la $1^{e}$ et la $2^{e}$ synthèse réalisées durant la phase de formation), sur les aspects pragmatique (prag.), cognitif (cog.) et langagier (lang.) des rédactions.

Les membres des groupes ne maintiennent cependant pas un meilleur niveau au post-test que les personnes s'étant formées en mode individuel tutoré. Le groupe tutoré semble ainsi être un moyen de production plus performant que la modalité individuelle tutorée, mais ne paraît pas induire pour autant un meilleur apprentissage.

Différents aspects inhérents à notre programme d'expérimentation peuvent participer à expliquer ce phénomène. Ainsi, les membres des groupes ont tendance à se répartir les sous-tâches en fonction de leurs compétences préexistantes, malgré les efforts du tuteur pour impliquer tous les membres dans chaque sous-tâche. Ce comportement, qui relève davantage de celui d'un groupe de production que de celui d'un groupe d'apprentissage (cf. Pléty 1998), favorise peu le développement de nouvelles compétences. Mais, travailler et apprendre en groupe fait appel à une compétence sociale qui ne peut pas automatiquement être considérée comme un pré-acquis (cf. Linard 2003). Autrement dit, l'interaction sociale aussi se construit et s'apprend.

Un autre biais possible est que notre expérimentation a comporté une formation de moyenne durée (trois mois environ), ce qui est relativement court pour une amélioration sensible et mesurable de compétences aussi générales que celles qui sont demandées lors de la rédaction d'une synthèse. Notre volonté a été d'expérimenter une formation portant sur une tâche complexe telle qu'elle est pratiquée en situation de formation réelle, et non une activité partielle et très ciblée qui aurait facilité l'obtention de résultats significatifs. L'absence de différences significatives entre le pré- et le post-test aurait de plus pu laisser penser que le manque d'évolution soit attribuable aux tests statistiques paramétriques utilisés, qui demandent des différences importantes pour les faibles effectifs que nous avions avant de les considérer comme significatives. L'observation de différences significatives en lien avec les deux synthèses rédigées durant la période de formation nous fait cependant dire que les tests statistiques ne sont que faiblement à remettre en cause dans notre recherche. Ces différents biais ont pu conditionner la faible présence de différences significatives observées. Il serait à vérifier, en variant les tests statistiques utilisés et le type de tâches si, dans d'autres contextes également, le groupe progresse davantage que chacun des individus qui le composent. 


\section{Conclusion}

En dépit des biais, nos résultats mettent en cause une pratique répandue dans formation à distance aujourd'hui : celle de l'évaluation individuelle qui fait suite à un apprentissage collectif en ligne. Le groupe restreint met du temps à se former et à bien fonctionner et il demande une certaine énergie aux apprenants pour l'organisation de l'action commune. Mais une fois formé, le groupe restreint tutoré permet de produire des rédactions de meilleure qualité que celles des personnes travaillant individuellement avec un tuteur. Il ne permet en revanche pas, au vu de nos résultats, d'améliorer davantage que le mode d'apprentissage individuel tutoré les savoir-faire individuels. Si les compétences visées (et l'évaluation) sont individuelles, l'interaction en groupe tutoré n'apparaît pas comme un moyen d'apprentissage plus performant que le mode individuel tutoré. L'évaluation de la co-action sociale ne correspond pas aux pratiques universitaires courantes et reste difficile et long à mettre en place. Mais lorsque l'objectif d'apprentissage n'est pas seulement le développement de compétences individuelles mais aussi sociales, l'évaluation de l'action conjointe s'impose. Ainsi, lorsque l'on se situe - comme le font aussi bien les dispositifs observés que le CLES - dans la perspective actionnelle en langues qui met en avant l'interaction sociale, l'évaluation ne devrait pas être individuelle, mais porter également sur la co-action sociale elle-même, c'est-à-dire sur le processus d'élaboration commune d'une réponse à une tâche donnée. Ce constat met en question la forme actuelle, essentiellement individuelle, de la certification en langues qui est en train de se mettre en place au niveau national.

\section{Bibliographie}

Bruner J.S. (1983). Le Développement de l'enfant: Savoir faire, savoir dire, Paris : Presses Universitaires de France, 1983, 292 p.

Chapelle, C.A. (2001). Computer Applications in Second Language Aquisition. Foundations for teaching, testing and research, Cambridge: Cambridge University Press, 215 p.

Conseil de l'Europe (2001). Cadre européen commun de référence pour les langues - apprendre, enseigner, évaluer, Paris : Didier, ( $3^{\mathrm{e}}$ édition), $192 \mathrm{p}$.

Demaizière, F. \& Dubuisson, C. (1992). De l'EAO aux NTF. Utiliser l'ordinateur pour la formation, Paris : Éditions Ophrys, 390 p.

Doise, W. \& Mugny, G. (1981). Le développement social de l'intelligence, Paris : InterEditions, 1981, $199 \mathrm{p}$.

Gilly, M. (1995). Approches socio-constructives du développement cognitif, In Gaonac'h D., Golder C. (coord.), Manuel de psychologie pour l'enseignement, Paris : Hachette Éducation, 130-167. 
Gilly, M. (2001). Interaction entre pairs et constructions cognitives : modèles explicatifs, in PerretClermont A.-N., Nicolet M. (dir.), Interagir et connaître. Enjeux et régulations sociales dans le développement cognitif, Paris : L'Harmattan, 2001, 20-32.

Henri, F \& Lundgren-Cayrol, K. (2001). Apprentissage collaboratif à distance. Pour comprendre et concevoir les environnement d'apprentissage virtuels, Sainte-Foy: Presses Universitaires du Québec, 2001, 184 p.

Linard, M. (2003). Apprendre à apprendre, Profession formateur, Amphis de la Cinquième, Émission no. 12 « Réorganiser la formation », diffusée sur TV5 le 2/4/03 (c 2000).

Ministère de l'Éducation Nationale (2001). Le certificat de compétences en langues pour l'enseignement supérieur, http://www.education.gouv.fr/sup/formation/cles.htm (dernière consultation : 12/03/04)

Nissen, E. (2003). Apprendre une langue en ligne dans une perspective actionnelle. Effets de l'interaction sociale, thèse nouveau régime, Strasbourg : Université Louis Pasteur, 321 p.

Nissen, E. (2004). Expérimentation et présupposés pédagogiques d'un dispositif d'enseignement des langues à distance intégrant le travail de groupe, Études de linguistique appliquée (éla), 134.

Panitz, T. (1997). Collaborative Versus Cooperative Learning, Cooperative Learning and College Teaching, V8, No. 2, Winter 1997, http://home.capecod.net/ tpanitz/tedsarticles/ coopdefinition.htm (dernière consultation: 12/03/04)

Perret-Clermont A.-N. (1996). La construction de l'intelligence dans l'interaction sociale, Bern, Berlin, Frankfurt/M., New York, Paris, Wien : Peter Lang, 1996 (c 1979), 305 p.

Pléty, R. (1998). Comment apprendre et se former en groupe, Paris, Retz, 143 p.

Poteaux N. (1998). Savoir raison garder, Ela. La didactique des langues en contexte scolaire, no. 111, juillet - septembre 1998, 315-324.

Puren C. (2002a). Innovation et cohérence didactique en langue, New Standpoints no. 12/2002, 3-7.

Puren C. (2002b). Perspectives actionnelles et perspectives culturelles en didactique des languescultures : vers une perspective co-actionnelle-co-culturelle, Les Langues modernes no. 3/2002, 55-70.

Rémigy, M.-J. (2001). Quand les désaccords favorisent l'apprentissage ?, Ruano Borbalan J.-Cl. (éd.), Éduquer et former, Paris : Éditions Sciences humaines, 2001 (2 édition revue, c 1997), 109-112.

Vygotsky L.S.(1985a). Pensée et langage, Paris : Éditions Sociales, 1985, 419 p.

Vygotsky L. S. (1985b). Le problème de l'enseignement et de développement mental à l'âge scolaire, in Schneuwly B., Bronckart J.P. (dir.), Vygotsky aujourd'hui, 1985, 95 - 117.

Warschauer, M. (1998). Interaction, Negotiation, and Computer-Mediated Learning, Clay M. (ed.), Practica Applications of Educational Technology in Language Learning, Lyon, INSA, 1998, http://www.insa-lyon.fr/Departements/CFRL/interaction.html (dernière consultation: 12/03/2004). 